\title{
Author Correction: Sequestration of T cells in bone marrow in the setting of glioblastoma and other intracranial tumors
}

\author{
Pakawat Chongsathidkiet (D), Christina Jackson, Shohei Koyama (D), Franziska Loebel, Xiuyu Cui, S. Harrison Farber (D), \\ Karolina Woroniecka, Aladine A. Elsamadicy, Cosette A. Dechant, Hanna R. Kemeny, Luis Sanchez-Perez, \\ Tooba A. Cheema, Nicholas C. Souders, James E. Herndon, Jean-Valery Coumans, Jeffrey I. Everitt, Brian V. Nahed, \\ John H. Sampson, Michael D. Gunn (D), Robert L. Martuza, Glenn Dranoff, William T. Curry and Peter E. Fecci
}

Correction to: Nature Medicine https://doi.org/10.1038/s41591-018-0135-2, published online 13 August 2018.

In the version of this article originally published, the figure callout in this sentence was incorrect: "Furthermore, in S1P1-KI mice themselves, whereas PD-1 blockade was ineffectual as monotherapy, the effects of 4-1BB agonism and checkpoint blockade proved additive, with the combination prolonging median survival and producing a 50\% long-term survival rate (Fig. $6 \mathrm{f}$ )." The callout should have been to Supplementary Fig. 6b. The error has been corrected in the PDF and HTML versions of the article.

\section{Publisher Correction: Myelodysplastic syndrome progression to acute myeloid leukemia at the stem cell level}

Jiahao Chen, Yun-Ruei Kao, Daqian Sun, Tihomira I. Todorova, David Reynolds, Swathi-Rao Narayanagari, Cristina Montagna, Britta Will, Amit Verma (i) and Ulrich SteidI(1)

Correction to: Nature Medicine https://doi.org/10.1038/s41591-018-0267-4, published online 03 December 2018.

In the version of this article originally published, Ulrich Steidl's name was listed as “and Ulrich Steidl." His name has been updated to "Ulrich Steidl." The error has been fixed in the print, PDF and HTML versions of this article.

Published online: 19 December 2018

https://doi.org/10.1038/s41591-018-0333-y

\section{Publisher Correction: ApoE attenuates unresolvable inflammation by complex formation with activated $\mathrm{Clq}$}

Changjun Yin (D), Susanne Ackermann (D), Zhe Ma, Sarajo K. Mohanta (D), Chuankai Zhang, Yuanfang Li, Sandor Nietzsche, Martin Westermann, Li Peng, Desheng Hu, Sai Vineela Bontha D , Prasad Srikakulapu, Michael Beer, Remco T. A. Megens, Sabine Steffens, Markus Hildner, Luke D. Halder, Hans-Henning Eckstein, Jaroslav Pelisek, Jochen Herms, Sigrun Roeber, Thomas Arzberger, Anna Borodovsky, Livia Habenicht, Christoph J. Binder, Christian Weber, Peter F. Zipfel ID, Christine Skerka (D) and Andreas J. R. Habenicht (iD

Correction to: Nature Medicine https://doi.org/10.1038/s41591-018-0336-8, published online 28 January 2019.

In the version of this article originally published, a sentence was erroneously included in the author contributions, and information regarding second shared authorship was missing from the author contributions. The following should not have been included in the author contributions: "C.W. and A.J.R.H. supervised the work presented in Figs. 1, 2, 5, 6; P.Z. and C.S. supervised the work presented in Figs. 3, 4." Additionally, this sentence should have appeared at the beginning of the author contributions: "These authors contributed equally: C.W., P.F.Z., C.S., and A.J.R.H.” The errors have been corrected in the print, PDF and HTML versions of the article. 